\title{
Uso de los organizadores gráficos interactivos en enseñanza de vocabulario básico de ingles
}

\section{Use of interactive graphic organizers in teaching basic English vocabulary}

Diana Carina Chicaiza Luisataxi. ${ }^{1}$, Carina Fernanda Vallejo Barreno. ${ }^{2}$ Keila Esther Guerrero Chacón. ${ }^{3}$, Holger Patricio Castillo Mazón. ${ }^{4}$ \& Diana Álvarez Rodríguez. ${ }^{5}$

DOI: https://doi.org/10.33262/concienciadigital.v3i3.1309

\begin{abstract}
.
The research aimed to analyze the use of the 3D interactive graphic organizers applied to teach basic English vocabulary to students of Ninth year of Basic General Education from the "Captain Edmundo Chiriboga" High School located in Riobamba-Ecuador. To this end, a qualitative, descriptive and field study was considered to be applied in an experimental group consisting of a sample of thirty-nine students among men and women with homogeneous knowledge of English. A planned teaching strategy was applied to the pilot group, which contains a set of activities based on interactive graphic organizers that were developed through expertise and advice from English peers of the English Department. To evaluate the outcome in learning the basic vocabulary, the survey technique was used, and as a tool a Likert-scale survey that was validated by experts in the area and submitted to Cronbach's Alpha. The results obtained were weighted, tabulated, analyzed and interpreted using statistical tables and graphs, which allowed to establish that the use of 3D interactive graphic organizers is an innovative teaching strategy that favors the teaching process learning of basic vocabulary of the English language.
\end{abstract}

Keywords: Usage, Graphic Organizers, Vocabulary, English, Interactive.

\footnotetext{
${ }^{1}$ Unidad Educativa Cap. Edmundo Chiriboga, Riobamba, Ecuador, ladydd4@ gmail.com

${ }^{2}$ Escuela Superior Politécnica de Chimborazo, Facultad de Administración de Empresas,

Riobamba, Ecuador, carina.vallejo@espoch.edu.ec

${ }^{3}$ Escuela Superior Politécnica de Chimborazo, Centro de Idiomas, Riobamba, Ecuador, carina.vallejo@espoch.edu.ec

${ }^{4}$ Escuela Superior Politécnica de Chimborazo, Facultad de Informática y Electrónica, Riobamba, Ecuador, holger.castillo@espoch.edu.ec

${ }^{5}$ Universidad Regional Autónoma de Los Andes, Centro de Transferencia y Desarrollo de Tecnología, Riobamba, Ecuador, dianaar870128@gmail.com
} 


\section{Resumen.}

La investigación tuvo como objetivo, analizar el uso de los organizadores gráficos interactivos 3D aplicados en el vocabulario básico del idioma inglés en estudiantes de Noveno Año de Educación General Básica de la Unidad Educativa Capitán Edmundo Chiriboga de la ciudad de Riobamba, Ecuador. Para ello, se consideró un estudio cualicuantitativo, descriptivo y de campo que fue aplicado en un grupo experimental conformado por una muestra de treinta y nueve estudiantes entre hombres y mujeres con conocimientos homogéneos de inglés. Al grupo experimental se aplicó una estrategia didáctica planificada, la cual contiene un conjunto de actividades basados en organizadores gráficos interactivos que fueron elaborados mediante con experticia y asesoramiento de pares del área de inglés. Para evaluar el resultado en el aprendizaje del vocabulario básico, se utilizó la técnica de la encuesta, y como instrumento una encuesta con escala de Likert que fue validado por expertos en el área y sometido al Alpha de Cronbach. Los resultados obtenidos fueron ponderados, tabulados, analizados e interpretados mediante tablas y gráficos estadísticos, los mismos que permitieron establecer que el uso de los organizadores gráficos interactivos 3D son una estrategia didáctica innovadoras que favorecen el proceso de enseñanza aprendizaje de vocabulario básico del idioma inglés.

Palabras clave: Uso, Organizadores gráficos, Vocabulario, Inglés, Interactivos.

\section{Introducción.}

El Con el tiempo en las clases de lengua extranjera en Ecuador, la enseñanza de vocabulario para el aprendizaje y la enseñanza de vocabulario han sido relegados. Erradamente se consideraba que una orientación meramente gramatical era principalmente necesaria. Se considera que a mayor profundidad del conocimiento del vocabulario facilita el proceso de aprendizaje del idioma inglés, ya que el repertorio de palabras ayuda al estudiante a entender con mayor facilidad diferentes interacciones con hablantes del idioma inglés.

Por lo que la presente investigación plantea a los organizadores gráficos interactivos enmarcado en los vocabularios como una estrategia didáctica innovadora para el proceso de enseñanza-aprendizaje del idioma inglés, desde una diferente perspectiva que facilita los aprendizajes de los estudiantes, ya que la información se organiza de tal manera que favorece la inducción de nuevos términos y conceptos,

La estrategia se enmarca en la organiza de la información para facilitar al educado a que capte hechos, nuevas palabras, teorías, conceptos, procesos e ideas que fomentan la creatividad, participación activa y retención a largo plazo de información.

Antes el conocimiento del vocabulario era una estrategia para la adquisición y aprendizaje de cualquier lengua. Lo que implica, qué sin constituir en primera instancia una sólida 
base de vocabulario, no se puede lograr comprensión y uso de un idioma. Por tanto, se propone que los docentes usen estrategias para enriquecer el nivel de conocimiento de vocabulario, mediante el uso de recursos didácticos que motiven y ayuden a los estudiantes en el aprendizaje significativo.

Según Córdova (2015) indica que se puede construir organizadores gráficos para ayudar a entender textos argumentativos, lograr interpretar, organizar y resumir información. Por otra parte, Boakes (2008) demostró que los organizadores gráficos interactivos contribuyen en el aprendizaje de la matemática mediante la utilización de los mismos números para la construcción de estos recursos, menciona además que los estudiantes mejoran la comprensión de términos y conceptos geométricos. Por otra parte, Robinson (1998) sugiere que solo se use los organizadores gráficos como sólo aquellos que se adapten al contenido.

García (2013), a través de instrumentos como la encuesta, entrevista y talleres, pudo evaluar y demostrar que con los organizadores gráficos se puede superar la deficiencia en la comprensión lectora, y obtener beneficios para en el aprendizaje.

Bloom (1956) es su obra “Taxonomía de los Objetivos de la Educación” establece los tres dominios del aprendizaje. Cada una de estas áreas permite al educando conocer, analizar, evaluar y poner en práctica lo aprendido, logrando así el dominio total de los contenidos.

Al utilizar los objetos de aprendizaje como estrategia didáctica para los estudiantes, Avanzini (1998) indica que una estrategia didáctica requiere de tres componentes básicos: misión, estructura curricular y retos cognoscitivos. Por su parte, Saturnino de la Torre (2002) indica que la elección de la estrategia adecuada, incide en el estudiante y en la manera de dictar la clase, en la institución y hasta la sociedad.

Herrera, Holmes, \& Kavimandan (2011) indican que al usar los organizadores gráficos como la estrategia, "permite a los profesores proveer a los estudiantes oportunidades estructuradas para practicar el lenguaje a través del escuchar, hablar, leer y escribir" (p. 64). Por lo que, el desarrollado de la clase debe contemplar tres etapas: Activación, Conexión y Afirmación. Es decir, primero se debe activar el conocimiento previo del estudiante sobre el nuevo contenido a tratarse. Las actividades que el maestro use permitirá al estudiante organizar sus primeras conceptualizaciones. Luego en la siguiente etapa de las conexiones, donde los organizadores gráficos ayudan a que el nuevo conocimiento sea plasmado en forma gráfica y conceptual con el fin de que se pueda hacer comparaciones o hallar similitudes con su conocimiento previo. Para que finalmente, afirmar el nuevo aprendizaje y ponerlos en práctica. Es decir, toda la información adquirida ayudará al estudiante a revisar y reforzar los nuevos conceptos, ideas, o teorías.

Prats (1996) sostiene para mejorar las habilidades intelectuales, y dominar los conocimientos adquiridos se necesitan recurso didáctico apropiados que deben ser 
utilizados por el estudiante y el maestro para apoyar los procesos mentales e interiorizar los conocimientos. Según Graells (2011), los recursos didácticos cumplen con diferentes funciones que están en sintonía de la necesidad y el propósito de su uso. Entre las funciones tenemos al recurso didáctico para proporcionar información valida y útil; para guiar los aprendizajes y para organizar la información, relacionar los nuevos conceptos, teoría o ideas, para crear nuevos conocimientos y aplicarlos en la vida real. Además, el recurso didáctico debe brindar oportunidades para ejercitar habilidades, motivar, despertar y mantener el interés del alumno en todas las etapas de la clase. Por otra parte, el recurso didáctico, debe evaluar los conocimientos, las habilidades que se tienen y deben proporcionar espacios para que el alumno exprese su imaginación.

\section{Metodología.}

La investigación se enmarcó en la modalidad socioeducativa, pues se fundamenta en aspectos metodológicos y principios teóricos, orientándose a la producción de conocimiento en educación, como un estudio que desea adaptarse o transformar el sistema social a través de contribuir para que la educación tradicional utilice alternativas como estrategias didácticas para mejorar el proceso de enseñanza aprendizaje.

El estudio tuvo enfoque cuali-cuantitativo, mediante investigación bibliográfica y descriptiva, que proporcionó la revisión previa de investigaciones mediante las teorías, hipótesis, experimentos, resultados, técnicas e instrumentos utilizados.

La investigación considera el análisis interpretativo de los resultados obtenidos, mediante la medición numérica y análisis estadístico de respuestas obtenidas de un cuestionario aplicado a la muestra investigada.

El diseño de la investigación fue cuasi-experimental, con la aplicación de un plan de intervención aplicado a un grupo experimental sin selección aleatoria o proceso de preselección.

En atención a lo planteado, la investigación determino el uso de los Organizadores Gráficos Interactivos 3D para el aprendizaje de vocabulario básico del idioma inglés, aplicado a estudiantes de Noveno Año de Educación Básica la Unidad Educativa Edmundo Chiriboga.

El instrumento de recolección de datos fue validado por tres expertos del área y fue aplicado a la muestra de la investigación, la misma que fue elaborada de forma estructurada y adaptada a los contenidos establecidos en el micro currículo.

La investigación fue de campo, pues se la desarrollo in situ donde se encuentra el objeto de estudio, es decir la Unidad Educativa "Edmundo Chiriboga" de la ciudad de Riobamba, permitiendo que el investigador procese la información de forma directa y segura. 
Se consideró una población integrada por estudiantes de los novenos años de Educación Básica de la Unidad Educativa “Cap. Edmundo Chiriboga G." de la ciudad de Riobamba provincia de Chimborazo durante el período 2016 - 2017, con un total de 76 estudiantes, con una edad promedio de entre 13 a 14 años de ambos sexos (femenino y masculino). De lo cual se tomó una muestra intencional de representada por paralelo "B" con 37, los mismos que fueron considerados como el grupo experimental.

La intervención aplicada a fin de obtener los resultados del uso de los Organizadores Gráficos Interactivos 3D en el vocabulario básico para el aprendizaje del idioma inglés, fue considerada como una estrategia didáctica la misma que se planifico en un periodo de duración de siete semanas.

Dentro de las dimensiones que se consideraron para el uso de los organizadores gráficos interactivos 3D, se consideraron: las habilidades de organización retención y producción. En los estilos de aprendizaje se contempló lo Visual y Kinestésico.

En el diseño para el uso de los organizadores se estableció: formatos básicos y aplicaciones; mientras que en el aprendizaje sus características, fases, condiciones y tipos.

La encuesta aplicada a la muestra de la investigación estaba compuesta por 10 preguntas cerradas con opciones de respuesta de selección; entre ellas: siempre, casi siempre, a veces, casi nunca y nunca. Los contenidos de las preguntas fueron realizados en función de los objetivos del estudio los mismos que fueron validados por tres expertos, quienes certificaron que las preguntas seleccionadas fueron claras y coherentes. Mientras que la confiabilidad fue a través de Alfa de Cronbach.

La aplicación de la encuesta, cuyo propósito fue obtener una perspectiva detallada sobre la opinión de los estudiantes con respecto al uso de los organizadores gráficos interactivos 3D. permitió que la información sea tabulada, la misma que fue organizada y procesada en términos de medidas descriptivas como son distribución de frecuencia, porcentajes.

El proceso que se realizó para procesar la investigación fue la siguiente:

- Cada pregunta estuvo condicionada mediante una escala de Likert (siempre, casi siempre, a veces, casi nunca, nunca)

- La información de la encuesta fue organizada y aplicada a los estudiantes mediante tablas de información.

- La información, fue ingresada en Microsoft Excel mediante tablas que permitió presentar los datos obtenidos mediante frecuencias y sus respectivos cálculos de porcentaje.

- Se realizó las representaciones gráficas de los datos obtenidos a través de gráficos de pastel, que facilitaron su comprensión y permitieron observar sus resultados. 
- Los datos obtenidos fueron analizaron en términos descriptivos, con la finalidad de interpretarlos y responder a los objetivos de la investigación.

- Finalmente, los datos fueron interpretados y permitieron establecer las conclusiones pertinentes.

\section{Resultados.}

Pregunta 1. Los Organizadores Gráficos Interactivos utilizados por la docente fueron una estrategia didáctica adecuada para el aprendizaje de nuevas palabras.

Tabla 1. OG como estrategia didáctica

\begin{tabular}{lcc}
\hline Alternativa & Frecuencia & Porcentaje \\
\hline Siempre & 23 & $59,0 \%$ \\
Casi Siempre & 11 & $28,2 \%$ \\
A Veces & 5 & $12,8 \%$ \\
Casi Nunca & 0 & $0,0 \%$ \\
Nunca & 0 & $0,0 \%$ \\
\multicolumn{1}{c}{ Total } & $\mathbf{3 9}$ & $\mathbf{1 0 0 \%}$ \\
\hline
\end{tabular}

Fuente: Elaboración propia.

Análisis e interpretación:

De los estudiantes encuestados, El 59,0\% indica que siempre los Organizadores Gráficos Interactivos utilizados por la docente fueron una estrategia didáctica adecuada para el aprendizaje de nuevas palabras, el 28,2\% indica que casi siempre; mientras que el 12,8\% indica que A veces. Por lo que la mayoría de los estudiantes encuestados considera que siempre los Organizadores Gráficos Interactivos utilizados por la docente fueron una estrategia didáctica adecuada para el aprendizaje de nuevas palabras. En consecuencia, los resultados representan una ventaja dentro de la presente investigación puesto que los Organizadores Gráficos Interactivos apoyaron tanto al docente en su planificación, como al estudiante en el aprendizaje de palabras, lo que indica que es conveniente que su uso sea más frecuente.

Pregunta 2. Los Organizadores Gráficos Interactivos ayudaron como un recurso o herramienta de estudio de palabras nuevas en Ingles. 
Tabla 2. OG y su ayuda como recurso

\begin{tabular}{lcc}
\hline Alternativa & Frecuencia & Porcentaje \\
\hline Siempre & 13 & $33,3 \%$ \\
Casi Siempre & 13 & $33,3 \%$ \\
A Veces & 8 & $20,5 \%$ \\
Casi Nunca & 3 & $7,7 \%$ \\
Nunca & 2 & $5,1 \%$ \\
$\quad$ Total & $\mathbf{3 9}$ & $\mathbf{1 0 0 \%}$ \\
\hline
\end{tabular}

Fuente: Elaboración propia.

Análisis e interpretación:

De los estudiantes encuestados el 33\% indica que siempre los Organizadores Gráficos Interactivos les ayudó como un recurso o herramienta de estudio de palabras nuevas en Ingles, el 33,3\% indica que casi siempre, el 20,5 indica que a veces, el 7,7\% indica que casi nunca y el $5,1 \%$ indica que nunca. La mayoría de los estudiantes encuestados considera que siempre los Organizadores Gráficos Interactivos les ayudó como un recurso o herramienta de estudio de palabras nuevas en Ingles. En consecuencia, los Organizadores Gráficos Interactivos pueden ser usados como una herramienta de estudio puesto que su capacidad de organización facilita la comprensión de contenidos, que pueden ser usados con el propósito de repasar, revisar, y estudiar las diferentes palabras tratadas en clase.

Pregunta 3. La utilización de Organizadores Gráficos Interactivos le ayudó a organizar y aprender, conceptos, vocabulario y nueva información en ingles de forma original.

Tabla 3. OG para la organización de concepto, vocabulario y nueva información

\begin{tabular}{lcc}
\hline Alternativa & Frecuencia & Porcentaje \\
\hline Siempre & 20 & $51,3 \%$ \\
Casi Siempre & 12 & $30,8 \%$ \\
A Veces & 6 & $15,4 \%$ \\
Casi Nunca & 1 & $2,6 \%$ \\
Nunca & 0 & $0,0 \%$ \\
\multicolumn{1}{c}{ Total } & $\mathbf{3 9}$ & $\mathbf{1 0 0 \%}$ \\
\hline
\end{tabular}

Fuente: Elaboración propia.

Análisis e interpretación:

De los estudiantes encuestados el 51,3\% indica que siempre la utilización de Organizadores Gráficos Interactivos, ayudó a organizar y aprender, conceptos, 
vocabulario y nueva información en ingles de forma original, el 30,8\% indica que casi siempre, el 15,4 indica que a veces y el 2,6\% indica que casi nunca. Es decir, la mayoría considera que siempre la utilización de Organizadores Gráficos Interactivos ayudo a organizar y aprender, conceptos, vocabulario y nueva información en inglés de forma original. De acuerdo a la opinión de los estudiantes esta herramienta apoya a la compresión y adquisición de nuevos conocimientos, en esta investigación es el caso del vocabulario.

Pregunta 4. La utilización de los Organizadores Gráficos Interactivos me ayudó a recordar el vocabulario aprendido durante la clase con mayor facilidad.

Tabla 4. OG para recordar vocabulario aprendido

\begin{tabular}{lcc}
\hline Alternativa & Frecuencia & Porcentaje \\
\hline Siempre & 22 & $56,4 \%$ \\
Casi Siempre & 12 & $30,8 \%$ \\
A Veces & 5 & $12,8 \%$ \\
Casi Nunca & 0 & $0,0 \%$ \\
Nunca & 0 & $0,0 \%$ \\
Total & $\mathbf{3 9}$ & $\mathbf{1 0 0 \%}$ \\
\hline
\end{tabular}

Fuente: Elaboración propia.

\section{Análisis e interpretación:}

De los estudiantes encuestados el 56,4\% indica que siempre la utilización de los Organizadores Gráficos Interactivos les ayudo a recordar el vocabulario aprendido durante la clase con mayor facilidad, el 30,8\% indica que casi siempre y el 12,8\% indica que a veces. Es decir, la mayoría de los estudiantes consideran el uso de los Organizadores Gráficos Interactivos, ayudo a recordar el vocabulario aprendido durante la clase con mayor facilidad.

La mente humana tiene la capacidad de retener un sinfín de información en diferentes etapas, empezado por la memoria sensorial, a corto plazo, y a largo plazo. Tratar de proporcionar al alumno las estrategias adecuadas que mejoren esta habilidad de retención es el objetivo de los organizadores gráficos interactivos. Al combinar información con imágenes, añadidas de práctica contrastante de los contenidos, ayuda a que un estudiante retenga lo que estudia en su cabeza, consecuentemente dicho conocimiento puede transformarse en un aprendizaje a largo plazo. De acuerdo a la percepción de los estudiantes, los Organizadores Gráficos Interactivos ayudaron a que su retención mejore, ya que pudieron recordar palabras con mayor facilidad.

Pregunta 5. Crear un organizador grafico interactivo ayudo a desarrollar mi creatividad y mejorar el proceso de aprendizaje de nuevas palabras en inglés. 
Tabla 5. OG para el desarrollo de creatividad y mejora de proceso de aprendizaje

\begin{tabular}{lcc}
\hline Alternativa & Frecuencia & Porcentaje \\
\hline Siempre & 32 & $82,1 \%$ \\
Casi Siempre & 7 & $17,9 \%$ \\
A Veces & 0 & $0,0 \%$ \\
Casi Nunca & 0 & $0,0 \%$ \\
Nunca & 0 & $0,0 \%$ \\
\multicolumn{1}{c}{ Total } & $\mathbf{3 9}$ & $\mathbf{1 0 0 \%}$ \\
\hline
\end{tabular}

Fuente: Elaboración propia.

Análisis e interpretación:

De los estudiantes encuestados, el 82,1\% indica que siempre crear un organizador grafico interactivo les ayudo a desarrollar la creatividad y mejorar el proceso de aprendizaje de nuevas palabras en inglés y el 17,9\% indica que casi siempre. Por lo que la mayoría de los estudiantes consideran que al crear un organizador grafico interactivo permite desarrollar la creatividad y mejorar el proceso de aprendizaje de nuevas palabras en inglés; es decir, que existe motivación para crear su propia herramienta de trabajo reflejados a través de la aplicación de colores, formas e incluso decoración de forma estructurada o pictórica para enfocarse en ideas clave, acceso fácil a la información y al estímulo del aprendizaje. Pregunta 6. Me agrada utilizar mis manos para crear un organizador gráfico interactvo y usar imágenes pues me facilita aprender nuevas palabras en inglés.

Tabla 6. OG con imágenes para el aprendizaje de nuevas palabras en inglés

\begin{tabular}{lcc}
\hline Alternativa & Frecuencia & Porcentaje \\
\hline Siempre & 33 & $84,6 \%$ \\
Casi Siempre & 4 & $10,3 \%$ \\
A Veces & 2 & $5,1 \%$ \\
Casi Nunca & 0 & $0,0 \%$ \\
Nunca & 0 & $0,0 \%$ \\
$\quad$ Total & $\mathbf{3 9}$ & $\mathbf{1 0 0 \%}$ \\
\hline
\end{tabular}

Fuente: Elaboración propia.

Análisis e Interpretación

De los estudiantes encuestados, el 84,6\% indican que siempre les agrada utilizar sus manos para crear un organizador gráfico y usar imágenes pues les facilita aprender nuevas palabras en inglés, el 10,3\% indica que casi siempre y el 5,1\% indica que a veces. Por lo que la mayoría considera que siempre les agrada utilizar sus manos para crear 
organizadores gráficos y usar imágenes ya que les facilita aprender nuevas palabras en inglés. Los estudiantes kinestésicos disfrutan el uso de Organizadores Gráficos Interactivos pues contienen actividad práctica para sus destrezas motrices, a través de doblar, cortar o pegar, que es opuesto a la usar hojas de trabajo o ejercicios de lápiz a papel; mientras que a los estudiantes visuales se les proporciona un recurso didáctico para ayudar a que su cerebro reciba y procese información a través de imágenes con información escrita, adquiriendo el conocimiento de manera mucho más efectiva.

Pregunta 7. Las aplicaciones de los diferentes Organizadores Gráficos Interactivos realizados en clases (mini book, tri-fold, endless chart, windows chart, etc. Me ayudaron a aprender no solo vocabulario sino a desarrollar otras destrezas.

Tabla 7. Aplicaciones de los organizadores gráficos

\begin{tabular}{lcc}
\hline Alternativa & Frecuencia & Porcentaje \\
\hline Siempre & 30 & $76,9 \%$ \\
Casi Siempre & 8 & $20,5 \%$ \\
A Veces & 1 & $2,6 \%$ \\
Casi Nunca & 0 & $0,0 \%$ \\
Nunca & 0 & $0,0 \%$ \\
\multicolumn{1}{c}{ Total } & $\mathbf{3 9}$ & $\mathbf{1 0 0 \%}$ \\
\hline
\end{tabular}

Fuente: Elaboración propia.

\section{Análisis e Interpretación}

De los estudiantes encuestados, el 76,9\% indican que siempre las aplicaciones de los diferentes Organizadores Gráficos Interactivos realizados en clases (mini book, tri-fold, endless chart, windows chart, etc. les ayudo a aprender no solo vocabulario sino a desarrollar otras destrezas, el 20,5\% indica que casi siempre y el 2,6\% indica que a veces. La mayoría consideran que las aplicaciones de los diferentes Organizadores Gráficos Interactivos les ayudo a aprender no solo vocabulario, sino también a desarrollar otras destrezas entre ellas el reading and writing, pues para ambas la importancia del vocabulario es vital, al utilizar los Organizadores Gráficos Interactivos como un recurso didáctico en el cual se registran palabras, conceptos o ideas, se está contribuyendo a que consecuentemente el estudiante mejore tanto su comprensión lectora, así como también el uso del idioma de forma escrita. De tal forma según esta percepción para los estudiantes cada uno de los Organizadores Gráficos Interactivos aplicados tiene su aporte en otras destrezas.

Pregunta 8. La información que escribo en los Organizadores Gráficos Interactivos me ayuda a conectar conocimientos previos con los nuevos. 
Tabla 8. OG para conectar conocimientos previos con los nuevos

\begin{tabular}{lcc}
\hline Alternativa & Frecuencia & Porcentaje \\
\hline Siempre & 21 & $53,8 \%$ \\
Casi Siempre & 17 & $43,6 \%$ \\
A Veces & 0 & $0,0 \%$ \\
Casi Nunca & 1 & $2,6 \%$ \\
Nunca & 0 & $0,0 \%$ \\
\multicolumn{1}{c}{ Total } & $\mathbf{3 9}$ & $\mathbf{1 0 0 \%}$ \\
\hline
\end{tabular}

Fuente: Elaboración propia.

\section{Análisis e Interpretación}

De los estudiantes encuestados, el 53,8\% indican que siempre la información que escribió en los Organizadores Gráficos Interactivos les ayudó a conectar conocimientos previos con los nuevos, el 43,6\% indica que casi siempre y el 2,6\% indica que casi nunca. Por lo que, la mayoría consideran que la información que escribió en los Organizadores Gráficos Interactivos ayudó a conectar conocimientos previos con los nuevos. Es decir, el aprendizaje de vocabulario no solo involucra conocer el significado de las palabras, también es importante saber cuándo usar estas palabras de acuerdo a los contextos presentados y que su uso sea de forma natural, pero además este conocimiento incluye la relación entre las palabras nuevas que se están aprendiendo y las ya adquiridas. Por tal motivo, los maestros deben usar estrategias que enseñen el significado de las palabras en contexto y ayudar a los estudiantes a asociar el nuevo vocabulario aprendido y lo que ya sabían. Según lo indican los estudiantes los Organizadores Gráficos Interactivos lograron crear esa conexión de conocimientos y permitieron afianzar e incrementar el número de palabras a su repertorio personal.

Pregunta 9. La utilización de los Organizadores Gráficos Interactivos me ayuda a aprender palabras nuevas a participar activamente en clase.

Tabla 9. OG para aprender palabras nuevas

\begin{tabular}{lcc}
\hline Alternativa & Frecuencia & Porcentaje \\
\hline Siempre & 13 & $33,3 \%$ \\
Casi Siempre & 21 & $53,8 \%$ \\
A Veces & 5 & $12,8 \%$ \\
Casi Nunca & 0 & $0,0 \%$ \\
Nunca & 0 & $0,0 \%$ \\
$\quad$ Total & 39 & $100 \%$ \\
\hline
\end{tabular}

Fuente: Elaboración propia. 


\section{Análisis e Interpretación}

De los estudiantes encuestados, el 53,8\% de los estudiantes indica que casi siempre la utilización de los Organizadores Gráficos Interactivos les ayudo a aprender palabras nuevas y a participar activamente en clase, el 33,3\% indica que siempre y el 12,8\% indica que a veces. La mayoría de los estudiantes encuestados considera que casi siempre la utilización de los Organizadores Gráficos Interactivos les ayudó a aprender palabras nuevas de una forma activa con el constante uso de las mismas y de su participación frecuente en clase. Uno de los objetivos del docente, es propiciar la participación activa en clase mediante estrategias que faciliten al estudiante se involucre. El aprendizaje de los estudiantes debe ser activo (escribir, leer, hablar y escuchar acerca del contenido que se esté tratando) y no pasiva a través de la participación de las actividades y no observando lo que se les explica.

Pregunta 10. Utilizar Organizadores Gráficos Interactivos me ayudó a aprender inglés de forma divertida y significativa.

Tabla 10. OG para aprender inglés de forma divertida y significativa

\begin{tabular}{lcc}
\hline Alternativa & Frecuencia & Porcentaje \\
\hline Siempre & 39 & $100,0 \%$ \\
Casi Siempre & 0 & $0,0 \%$ \\
A Veces & 0 & $0,0 \%$ \\
Casi Nunca & 0 & $0,0 \%$ \\
Nunca & 0 & $0,0 \%$ \\
$\quad$ Total & $\mathbf{3 9}$ & $\mathbf{1 0 0 \%}$ \\
\hline
\end{tabular}

Fuente: Elaboración propia.

\section{Análisis e Interpretación}

De los estudiantes encuestados, el 100,0\% de los estudiantes indica que siempre utilizar Organizadores Gráficos Interactivos les ayudó a aprender inglés de forma divertida y significativa.

\section{Conclusiones:}

- El empleo de las herramientas de estudio innovadoras permite que el estudio sea menos pesado, más gratificante y por supuesto que conlleve menos tiempo y esfuerzo al obtener resultados. Facilitar y sintetizar los contenidos mediante los organizadores gráficos interactivos produce el aumento de la motivación hacia el estudio, quedando finalmente reflejado en unos resultados efectivos.

- A través del material didáctico o pedagógico elaborado, fue diseñado para superar el conocimiento memorístico general y tradicional de las aulas y lograr un 
aprendizaje más integrador, comprensivo, de largo plazo, autónomo y estimulante. Al Involucrar al estudiante en la construcción de su propias definiciones e interpretaciones de las palabras nuevas. Los organizadores gráficos interactivos al ser una combinación de elementos lingüísticos (palabras, frases, e imágenes) permitiendo enormemente que el estudiante alcance un aprendizaje significativo.

- La percepción de estudiante indica que existe agrado por crear su propia herramienta de trabajo a través de la personalización con colores, formas, e incluso la decoración que el estudiante plasma en sus trabajos; es decir, los motivan a trabajar con herramientas para la presentación de información de una forma estructurada o pictórica. Además, ayuda a los estudiantes a enfocarse en sus ideas principales, acceso fácil a la información y estímulo del aprendizaje; es decir, los Organizadores Gráficos Interactivos se convierten estrategias creativas nuevas, porque estimulan al estudiante y al maestro en su proceso creativo.

\section{Referencias bibliográficas:}

Avanzini, G. (1998). La pedagogía hoy. México: Fondo de Cultura Económica.

Bloom, B. (1956). Taxonomy of educational objectives. Book 1 Cognitive Domain. New York: David McKay \& Co.

Boakes, N. (2008). Origami Mathematics Lessons: Paper folding as a teaching tool.

Córdova, M. E. (2012). Organizadores visuales y niveles de comprensión lectora de los alumnos de secundaria de la I.E. República Federal de Alemania, Puente Piedra 2012. Recuperado de http://cybertesis.unmsm.edu.pe/handle/cybertesis/4415

García, E. J. (2013). Los organizadores gráficos una poderosa herramienta de enseñanza y aprendizaje en el quinto año de la carrera de lengua y literatura, durante el II semestre del año lectivo 2012 y I semestre del año 2013. Recuperado de http://riul.unanleon.edu. ni:8080/jspui/bitstream/123456789/3231/1/225847. pdf

Graells, P. M. (7 de 8 de 2011). Los Medios Didácticos. Recuperado el 11 de Agosto de 2017, de http://peremarques.pangea.org/medios.htm

Herrera, S., Holmes, M., \& Kavimandan, S. (2011). Strategy Foldables. En S. Herrera, M. Holmes, \& S. Kavimandan, Crossing the Vocabulary Bridge: Differentiated Strategies for Diverse Secondary Classrooms (págs. 64-70). New York: Teachers College Press.

Prats, J. (1996). El nuevo modelo curricular y la elección del libro de texto. El bribo de texto y materiales didácticos (págs. 71-86). Madrid: Universidad Complutense de Madrid. 
Robinson, D. A. (1998). Recopilación de Organizadores gráficos ideales para primaria PROBLEMA ACCIÓN RESULTADO. Recuperado de https://www.orientacionandujar.es/2013/11/04/ recopilacion-de-organizadoresgraficos-idealespara-primaria-problema-accion-resultado

Torre, S. d. (2002). Estrategias didácticas innovadoras: recursos para la formación y el cambio. En O. B. Saturnino de la Torre, Estrategias didácticas innovadoras (pág. 108). Octaedro, S.L. 


\section{PARA CITAR EL ARTÍCULO INDEXADO.}

Chicaiza Luisataxi, D. C., Vallejo Barreno, C. F., Guerrero Chacón, K. E., Castillo Mazón, H. P., \& Álvarez Rodríguez, D. (2020). Uso de los organizadores gráficos interactivos en enseñanza de vocabulario básico de ingles. ConcienciaDigital, 3(3), 260274. https://doi.org/10.33262/concienciadigital.v3i3.1309

\section{【 Clencia}

El artículo que se publica es de exclusiva responsabilidad de los autores y no necesariamente reflejan el pensamiento de la Revista Ciencia Digital.

El artículo queda en propiedad de la revista y, por tanto, su publicación parcial y/o total en otro medio tiene que ser autorizado por el director de la Revista Ciencia Digital.
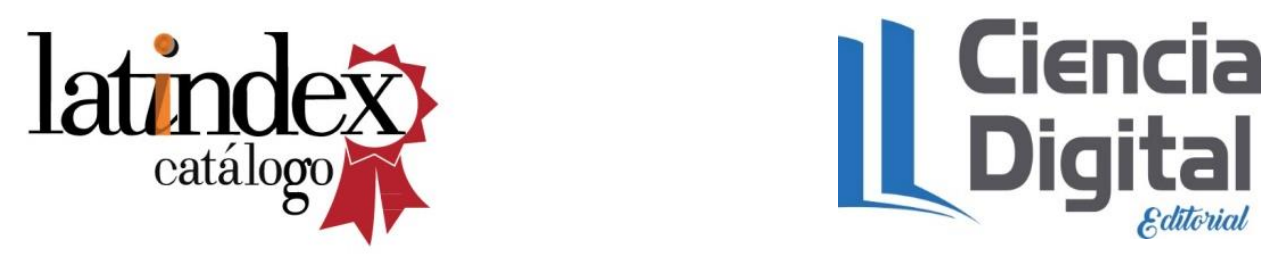